\title{
Microwave Dielectric Properties of CCTO/PVA Composites
}

\author{
M. P. F. Graça, ${ }^{1}$ K. D. A. Sabóia, ${ }^{2,3}$ F. Amaral $\mathbb{D}^{1,4}$ and L. C. Costa $\mathbb{D}^{1}$ \\ ${ }^{1}$ I3N and Physics Department, University of Aveiro, 3810-193 Aveiro, Portugal \\ ${ }^{2}$ Universidade Estadual do Ceará, Fortaleza, Brazil \\ ${ }^{3}$ Laboratório de Telecomunicações e Ciência e Engenharia de Materiais-LOCEM, Departamento de Física, \\ Universidade Federal do Ceará, Fortaleza, Brazil \\ ${ }^{4}$ Coimbra Health School, Polytechnic Institute of Coimbra, Coimbra, Portugal
}

Correspondence should be addressed to F. Amaral; filipe.amaral79@hotmail.com

Received 13 July 2018; Revised 28 September 2018; Accepted 10 October 2018; Published 2 December 2018

Academic Editor: Fernando Rubio-Marcos

Copyright ( 12018 M. P. F. Graça et al. This is an open access article distributed under the Creative Commons Attribution License, which permits unrestricted use, distribution, and reproduction in any medium, provided the original work is properly cited.

The $\mathrm{CaCu}_{3} \mathrm{Ti}_{4} \mathrm{O}_{12}$ (CCTO) ceramic powder was inserted in the polyvinyl alcohol (PVA) polymeric matrix, with an increasing weight fraction of the filler, to form a flexible and high dielectric constant composite at the $\mathrm{GHz}$ region. The structural characterization of the samples was performed using X-ray diffraction and scanning electron microscopy (SEM). The complex permittivity was calculated by the small perturbation theory using two resonant cavities $(2.7 \mathrm{GHz}$ and $5.0 \mathrm{GHz})$. Several classical models (Maxwell Garnett, Lichtenecker, effective medium theory (EMT), and Yamada) were used to fit the real part of the complex permittivity of the composite as a function of the weight fraction of CCTO powder inserted in the PVA matrix. The best predictions for the dielectric behavior of these samples were obtained with the EMT and Yamada models.

\section{Introduction}

Materials with excellent dielectric properties, such as a highdielectric constant, low dissipation factor, and good stability over a broad frequency and temperature ranges, are required for some of modern electronic applications. In the last few years, it has emerged an increasing interest in size reduction and performance enhancement of embedded passive components, especially for embedded capacitors [1-3]. Materials for such kind of applications must present (beyond the already mentioned properties) good dielectric and thermal stability over a broad frequency and temperature ranges. Furthermore, the final piece should be manufactured in a simple way, with low production costs. To achieve this goal, many trials were performed with ceramic-polymer composites with high-dielectric constant [4-7].

Generally, ceramics with high-dielectric constant values are very promising for capacitive applications in microelectronics and microwave devices (like dielectric resonators for cell mobile phones, for example) where miniaturization is crucial. However, it is well known that, for some applications, they are too brittle which becomes a critical factor.
On the contrary, polymers that have normally lower dielectric constant, but present a much lower dielectric loss, are flexible, easy to process, and traditionally used in lowleakage capacitors. The association of high-dielectric constant ceramics with a polymer results in composites with acceptable dielectric constant and loss factor values, with lower dependence on frequency and temperature and, simultaneously, more flexible than ceramics [5-7].

In this work, the polyvinyl alcohol (PVA) was used as a polymeric matrix, and $\mathrm{CaCu}_{3} \mathrm{Ti}_{4} \mathrm{O}_{12}$ (CCTO) ceramic particles were used as a filler. PVA is a water-soluble polymer widely applied in adhesives, sealants, coatings, paints, plastics, textiles, etc. It is also a polymer of great interest because of its characteristics, for various pharmaceutical and biomedical applications. PVA is produced by polymerization of vinyl acetate to polyvinyl acetate (PVAc), followed by hydrolysis of PVAc to PVA. Because the hydrolysis reaction does not go to completion, the final result of the polymerization process is a copolymer of PVA and PVAc. The PVA is normally supplied in powder form, and several grades are available with varying viscosity and solubility characteristics [8]. Besides the simplicity associated with the 
PVA synthesis process, we have chosen this polymer matrix because of its high thermal and chemical stabilities and nontoxicity. PVA also presents a high-dielectric strength and good charge storage capacity, which makes it usable as the polymer matrix in composite-based electronic components $[9,10]$.

On the contrary, $\mathrm{CaCu}_{3} \mathrm{Ti}_{4} \mathrm{O}_{12}$ (CCTO) is a known oxide of the $\mathrm{ACu}_{3} \mathrm{M}_{4} \mathrm{O}_{12}$ family. Subramanian et al. have been the first to report the exceptional dielectric properties of CCTO [11], and since then, a significant effort has been devoted to understand the polarization mechanisms that justify the colossal dielectric constant $\left(\varepsilon^{\prime}>10^{5}\right)$ observed for poly$[12,13]$ and single-crystalline ceramics [14]. The CCTO is a perovskite-like compound that, depending on the synthesis and sintering process, can present a dielectric constant (at $1 \mathrm{kHz}$ and room temperature) as high as $10^{5}$ and such dielectric properties are almost temperature- and frequencyindependent for large ranges [15-17]. The CCTO has a high potential for technological applications, like memory devices based on capacitive components [18].

Many CCTO/polymer composites have been investigated in the last years $[6,19,20]$. Some of them, CCTO/ epoxy and CCTO/polyvinylidene fluoride, for instance, are very promising to be used as new embedded capacitors $[19,21-23]$. The dielectric properties of such composites are, however, very dependent on temperature, and the dissipation factor is high. Besides, their preparation process is complex and harsh thermal environments are necessary to improve their thermal properties.

Rarely the dielectric response of CCTO/polymers at the microwave frequency region has been reported. Considering this lack, this paper is focused on the development of a new kind of CCTO/polymer composite prepared by a simple method and with desirable dielectric properties, particularly at the microwave frequency region, such as a high-dielectric constant and low dissipation factor. The complex permittivity of the composites was calculated by the small perturbation theory using two resonant cavities, at $2.7 \mathrm{GHz}$ and 5.0 GHz. Several classical models (Maxwell Garnett, Lichtenecker, effective medium theory (EMT), and Yamada) were used to fit the real part of the complex permittivity of the composite as a function of the weight of CCTO powder inserted in the PVA matrix.

\section{Experimental Methods}

2.1. Sample Preparation. The CCTO powder was prepared by the solid-state method using stoichiometric quantities of calcium carbonate $\left(\mathrm{CaCO}_{3}\right)$ (Aldrich, 99\%), titanium oxide $\left(\mathrm{TiO}_{2}\right)$ (Aldrich, 99\%), and copper (II) oxide (CuO) (Merck). The powders were mixed in a Fritsch 7.0 planetary mill for $30 \mathrm{~min}$ at $370 \mathrm{rpm}$. Milling was performed in agate vials and balls, under air, with a powder/balls weight fraction of $1: 9$. Therefore, the milling was used to guarantee the homogeneity of the powder. Then, the mixed powder was submitted to calcination in air at about $1,320 \mathrm{~K}$ for $24 \mathrm{~h}[24,25]$. The PVA (polyvinyl alcohol [- $\left.\mathrm{CH}_{2} \mathrm{CHOH}-\right]_{n}$, from Merck) flakes were milled in a Fritsch 7.0 planetary mill to obtain a powder with granulometry lower than $200 \mu \mathrm{m}$. The X-ray analysis confirmed the presence of CCTO as the major phase. The CCTO powder was added to PVA, to obtain composites with weight fraction between 0 and $70 \%$ to study the dielectric response in the microwave region. The mix of CCTO and PVA was milled for $100 \mathrm{~min}$, and the resulting material was uniaxially pressed into small cylinders, with $15 \mathrm{~mm}$ in height and $2 \mathrm{~mm}$ in diameter, applying $4.0 \mathrm{MPa}$ during $20 \mathrm{~min}$. These cylindrical samples were submitted to a heat treatment of about $420 \mathrm{~K}$ for two hours.

Composites with a weight fraction higher than $70 \%$ produced brittle samples; thereafter, their use as an electronic component would be compromised. We also point out that the preparation method used in this work to produce the CCTO/PVA composites presents some advantages compared to the wet techniques. In particular, the maximum weight fraction that is admitted for CCTO/PVA composites prepared by the wet techniques is about $30 \%$, which, along with a more complex procedure, makes our method suitable for future industrial applications [26, 27].

The adopted nomenclature to point the weight fraction of the composites is $\operatorname{CCTO}(x)$, where $x$ stands for the percentage of CCTO in the sample. For instance, CCTO(30) represents a sample with $70 \%$ (weight) of PVA and $30 \%$ (weight) of CCTO.

2.2. Structural and Morphological Characterization. The structure of the obtained samples was examined by X-ray diffraction using a X'Pert MPD Philips diffractometer (CuK $\alpha$ radiation, $\lambda=1.54056 \AA$ ) at $40 \mathrm{kV}$, and $30 \mathrm{~mA}$, with a curved graphite monochromator, an automatic divergence slit, a progressive receiving slit, and a flat plane sample holder in a Bragg-Brentano parafocusing configuration with a step of $0.04^{\circ}$ in $1 \mathrm{~s}$, in the $2 \theta$ angle range of $10-60^{\circ}$. The samples morphology was characterized by scanning electron microscopy (SEM) using a Hitachi S4100. To perform this analysis, all the samples were covered with carbon.

2.3. Dielectric Measurements. To study the dielectric response of the composites in the high-frequency region, we used the resonant cavity method. The measurement of the complex permittivity was performed using two resonant cavities $(2.7 \mathrm{GHz}$ and $5.0 \mathrm{GHz})$ [28]. When the sample is introduced inside the cavity, a perturbation of the electromagnetic field is induced. As a consequence, there is a change in the transmission in the cavity, shifting the resonance frequency, and degrading the quality factor. The shift in the cavity resonant frequency, $\Delta f$ (Figure 1), is related to the real part of the complex permittivity, $\mathcal{\varepsilon}^{\prime}$, while the change on the inverse of the quality factor of the cavity, $\Delta(1 / Q)$, is related to the imaginary part of the complex permittivity, $\varepsilon^{\prime \prime}$. It is possible to obtain a simple expression when we consider only the first-order perturbation in the electric field caused by the sample [29]:

$$
\frac{\Delta f}{f_{0}}+i \Delta\left(\frac{1}{2 Q}\right)=\varepsilon_{0}\left(\varepsilon^{*}-1\right) \frac{\int_{V_{s}} E_{i}^{*} E_{0} d V}{\int_{V} \varepsilon_{0} E_{0}^{2} d V},
$$




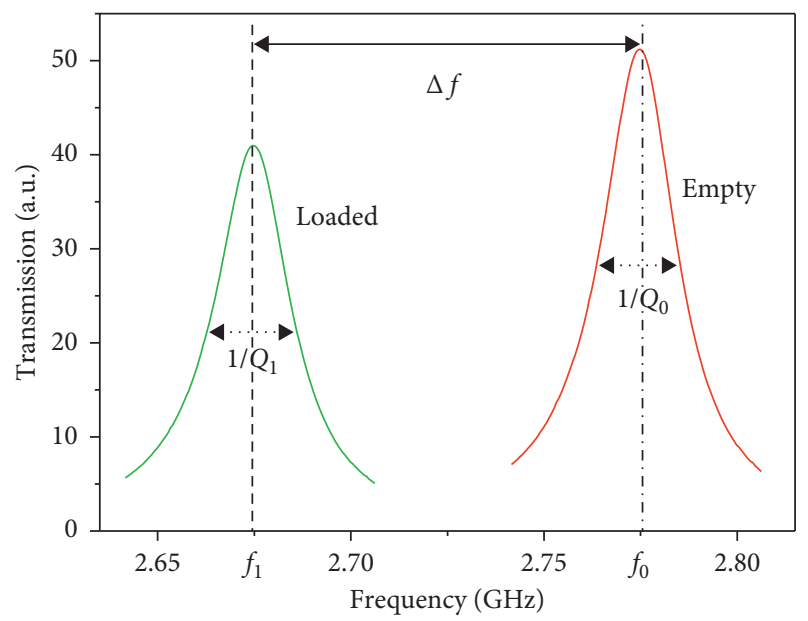

FIgUre 1: Transmission of the cavity with and without PTFE samples, showing the two resonances separated by $\Delta f$.

where $f_{0}$ is the resonance frequency of the cavity, $\varepsilon^{*}=$ $\varepsilon^{\prime}-i \varepsilon^{\prime \prime}$ is the complex permittivity of the material, and $E_{i}$ and $E_{0}$ are the electric fields inside and outside the material, respectively. The integration is performed in the volume of the sample, $V_{\mathrm{S}}$, and in the volume of the cavity, $V$. Splitting the real and imaginary parts, we can obtain the expressions for $\varepsilon^{\prime}$ and $\varepsilon^{\prime \prime}$ :

$$
\begin{aligned}
& \varepsilon^{\prime}=K \frac{\Delta f}{f_{0}} \frac{V}{V_{\mathrm{S}}}+1, \\
& \varepsilon^{\prime \prime}=\frac{K}{2} \Delta\left(\frac{1}{Q}\right) \frac{V}{V_{\mathrm{S}}},
\end{aligned}
$$

where $K$ is a constant related to the depolarization factor, which depends upon the geometric parameters. This parameter can be determined using a sample of known dielectric constant. In our case, we used a polytetrafluorethylene (PTFE) sample with the same size and shape of the studied samples. In these measurements, were used rectangular cavities operating in the $\mathrm{TE}_{1,0,5}$ and $\mathrm{TE}_{1,0,11}$ modes with the resonant frequencies of $2.7 \mathrm{GHz}$ and $5.0 \mathrm{GHz}$, respectively. The transmission of the cavities was measured using a HP 8753D Network Analyzer, at room temperature.

\section{Results and Discussion}

3.1. X-Ray Analysis. The XRD patterns were obtained at room temperature ( $300 \mathrm{~K}$ ) by step scanning using powdered samples. Figure 2 shows the XRD pattern of the base samples (CCTO and PVA) and of the CCTO $(x)$ for $x=30$ and $x=50$. The pattern obtained from the database of Inorganic Crystal Structures Database (ICSD) confirms the presence of CCTO as a major phase, presenting a cubic structure, with space group $1 \mathrm{~m}-3 \mathrm{~m}$ and space group number 229. Using Celref program [30], the lattice parameters of the CCTO powders were obtained: $a=b=c=7.3998 \pm 0.0027 \AA$ and $\alpha=\beta=\gamma=$ $90.00^{\circ}$, which is in accordance with the ICSD data of CCTO $\left(a=7.3930 \AA\right.$ and $\left.\alpha=\beta=\gamma=90.0000^{\circ}\right)$. From the patterns of

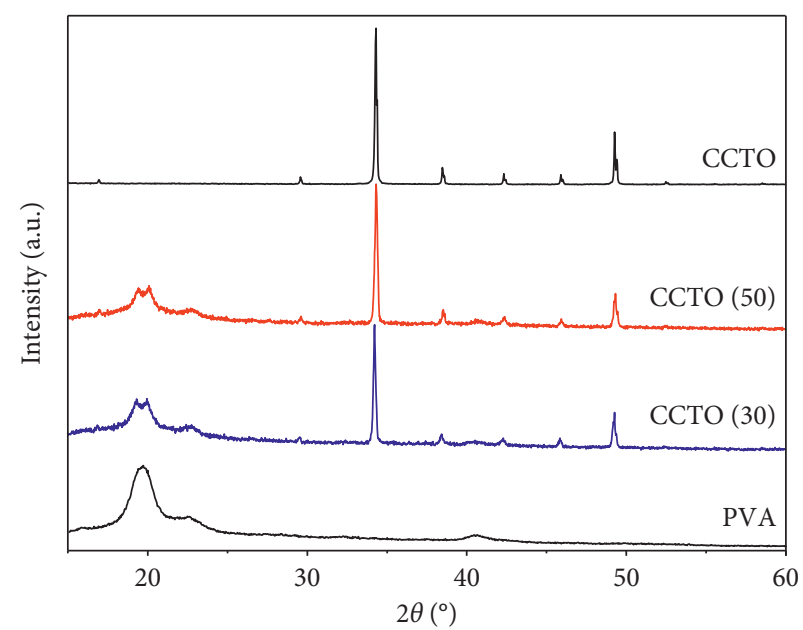

FIgure 2: XRD patterns of the CCTO, PVA, and CCTO $(x)$ for $x=$ 50 and 30 .

the base samples, we could see in Figure 2 that the patterns of the samples with $x=30$ and $x=50$, for example, are formed by peaks that can only be associated with PVA and CCTO. Although it is not presented here, the samples with different values of $x$ show the same pattern. This result was already expected, once the temperature that we used for the heat treatment of the cylindrical samples is too low to degrade the polymer or to initiate any chemical reaction between the CCTO and the polymer.

3.2. SEM Analysis. Figure 3 shows the micrographs of some of the prepared composites. In Figure 3(a), we present the micrograph of the PVA sample. From the micrograph of the surface of PVA polymer sample, we can identify large polymer platelets. Between these structures, it is possible to identify many holes uniformly distributed throughout the sample. Figure 3(b) shows the micrograph of the CCTO particles. From this micrograph, it is possible to assume that the CCTO grains have dimensions ranging between $3 \mu \mathrm{m}$ and $15 \mu \mathrm{m}$, presenting a spherical-like shape.

Figure 3(c) shows the micrograph of the $\mathrm{CCTO}(20)$ sample, and Figure 3(d) shows the micrograph of the CCTO(50) sample, both with an amplification of 100 times. From the analysis of Figures 3(c) and 3(d), we can point that the porosity is low, particularly for the higher CCTO percentage $(\mathrm{CCTO}(50))$. Combining these micrographs with those with higher amplification (x1k), we can state that, in both cases, the polymeric phase completely enfolds the ceramic particles, which means that a good affinity between these two phases is expected. The polymer is responsible for the aggregation of the ceramic powder and for the stiffness of the final composite, keeping the piece with enough mechanical strength for technological applications.

3.3. Dielectric Analysis. Figure 4 presents the signal transmission along the $2.7 \mathrm{GHz}$ resonant cavity for the following samples: PVA, $\operatorname{CCTO}(x)$ (for $x=10,20,30,40$ ), PTFE, and empty cavity. The observed curves of the transmission for 


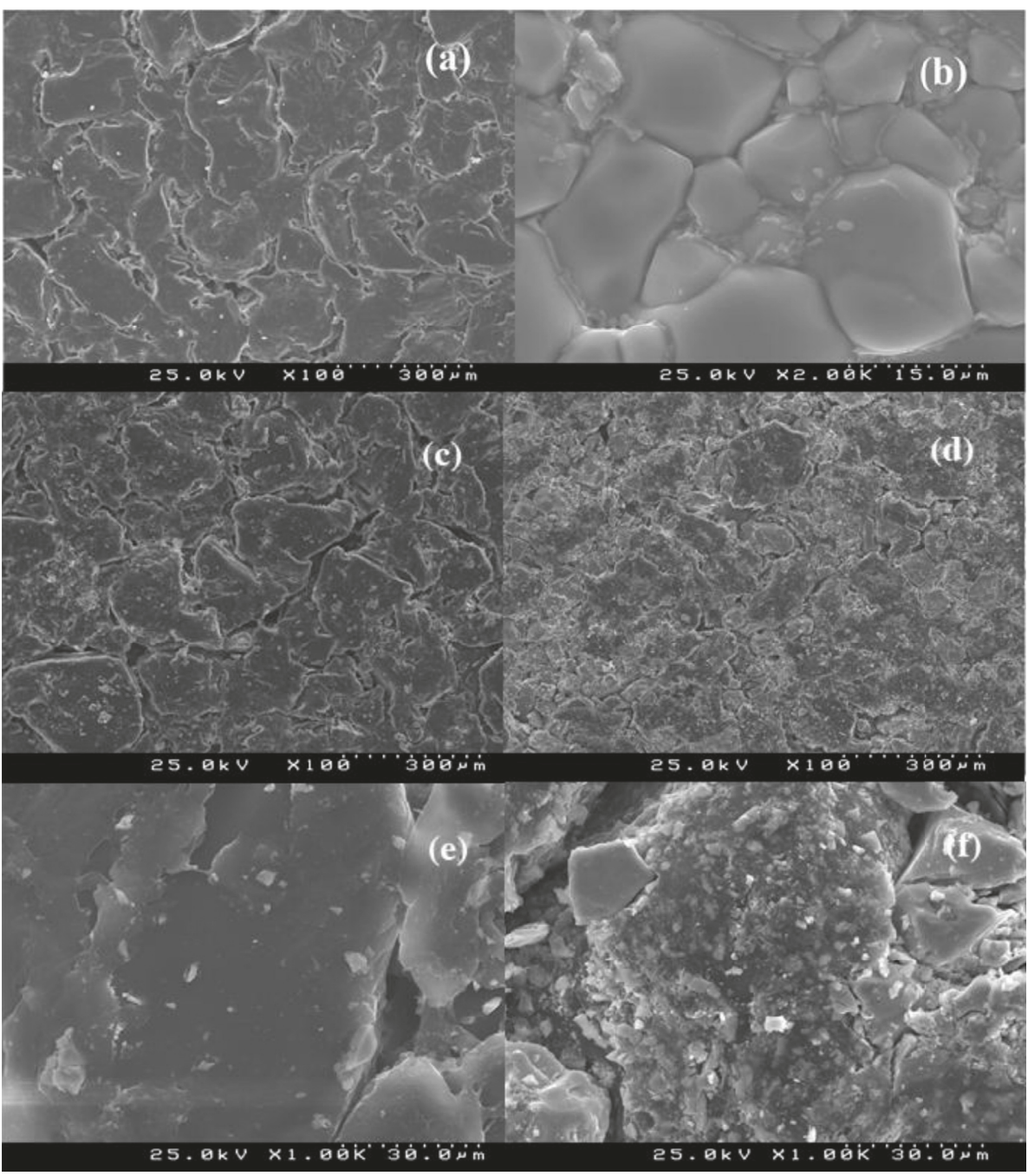

Figure 3: SEM micrographs of (a) PVA sample (100x), (b) CCTO powders (2000x), (c) CCTO(20) (100x), (d) CCTO(50) (100x), (e) $\operatorname{CCTO}(20)(1000 x)$, and (f) CCTO(50) (1000x).

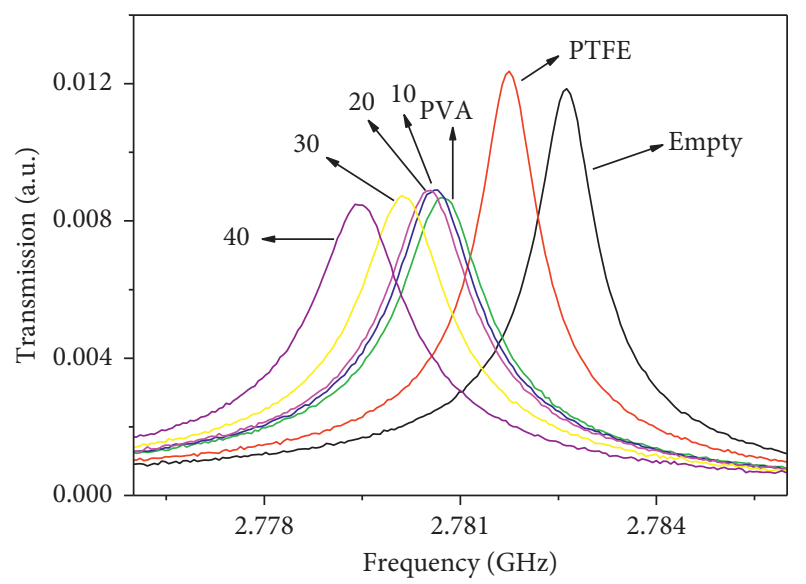

FIgURE 4: Transmission of the $2.7 \mathrm{GHz}$ resonant cavity for PVA, $\operatorname{CCTO}(x)$ (for $x=10,20,30,40)$, PTFE, and empty cavity.

the samples with $x=50,60$, and 70 are very close to the values found for $x=40$. These are not presented to avoid overlapping of the curves. Increasing the weight fraction of
CCTO powder of the composite implies a larger perturbation in the electromagnetic field configuration, leading to a frequency shift to the lower frequencies (red shift) and a pronounced attenuation of the intensity of the transmission signal.

Figure 5 shows the dielectric constant, as a function of the weight fraction of CCTO in the composites, for $2.7 \mathrm{GHz}$ and $5.0 \mathrm{GHz}$ cavities, measured at room temperature. It also shows the imaginary part of the complex permittivity $\left(\varepsilon^{\prime \prime}\right)$ as a function of the weight fraction of CCTO for the $5.0 \mathrm{GHz}$ cavity. The values of $\varepsilon^{\prime \prime}$ in the cavity of $2.7 \mathrm{GHz}$ were the same for all the samples which means that $\varepsilon^{\prime \prime}$ is too low to be measured by this method. As expected, the dielectric constant $\left(\varepsilon^{\prime}\right)$ increases with the weight fraction of CCTO, for each of the analyzed frequencies, and a similar behavior is observed for $\varepsilon^{\prime \prime}$. The real part of the complex permittivity for the pure PVA samples was 5.5 at $2.7 \mathrm{GHz}$ and 5.3 at $5.0 \mathrm{GHz}$ (and $\left.\varepsilon^{\prime \prime}=0.11\right)$. For the sample CCTO(10), it was measured 7.4 at $2.7 \mathrm{GHz}$ cavity and 6.7 at $5.0 \mathrm{GHZ}\left(\varepsilon^{\prime \prime}=0.11\right)$. The dielectric constants for the sample $\operatorname{CCTO}(70)$ were 17.4 at $2.7 \mathrm{GHz}$ and 16.2 at $5.0 \mathrm{GHZ}\left(\varepsilon^{\prime \prime}=0.31\right)$. 


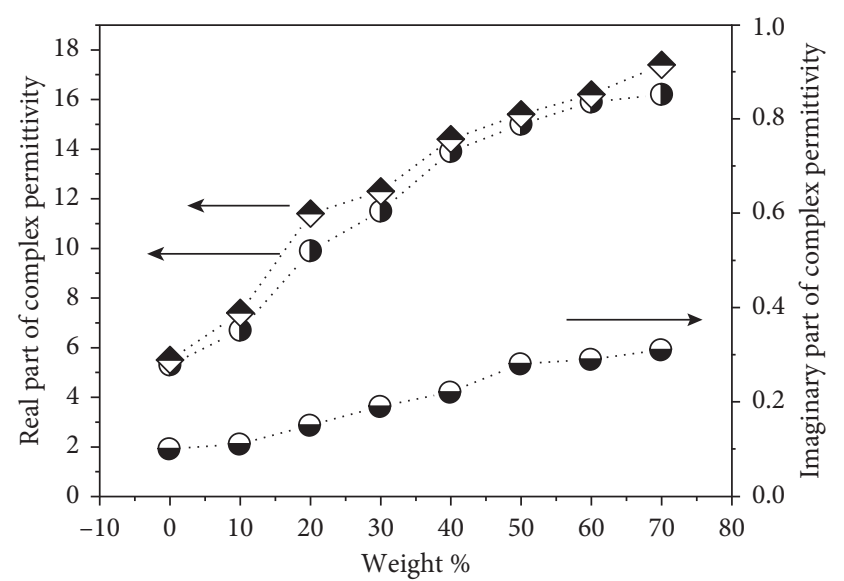

$$
\begin{aligned}
& \text { ๑. } 5 \mathrm{GHz} \\
& \text { ๑. } 2.7 \mathrm{GHz} \\
& \text { ๑. } 5 \mathrm{GHz}
\end{aligned}
$$

FIGURE 5: Real and imaginary parts of complex permittivity measured in $2.7 \mathrm{GHz}$ and $5 \mathrm{GHz}$ resonant cavity, for different weight fractions of CCTO.

Several models were used in order to predict the dielectric constant of the composite material. Figure 6 shows the experimental dielectric constant at $5.0 \mathrm{GHz}$, and, for comparison, the dielectric constants predicted by the Maxwell Garnett, Lichtenecker, EMT, and Yamada models are also included. The Maxwell Garnett [31] and the Lichtenecker $[32,33]$ models describe the dielectric property of a biphasic dielectric mixture comprising spherical crystallites, with high-dielectric constant, dispersed in a matrix of lowdielectric constant. According to the Maxwell Garnett model, the effective dielectric constant of the composite is given by

$$
\begin{aligned}
& \varepsilon^{\prime}=\varepsilon_{1}\left(1+\frac{3 V_{\mathrm{f}} \beta}{1-V_{\mathrm{f}} \beta}\right), \\
& \beta=\frac{\varepsilon_{2}-\varepsilon_{1}}{\varepsilon_{2}+2 \varepsilon_{1}},
\end{aligned}
$$

where $\varepsilon_{1}$ and $\varepsilon_{2}$ are the permittivities of the polymer matrix and of the CCTO, respectively. $V_{\mathrm{f}}$ is the volume fraction of the ceramic fillers. The Lichtenecker model is expressed by

$$
\ln \varepsilon^{\prime}=V_{\mathrm{f}} \ln \varepsilon_{2}+\left(1-V_{\mathrm{f}}\right) \ln \varepsilon_{1} .
$$

From Figure 6, one can see that the predicted values for $\varepsilon^{\prime}$, for both Maxwell Garnett and Lichtenecker models, deviate too much from that of the measured experimental values, only correctly fitting for the samples with lower (10\%) and higher $(70 \%)$ weight fractions of CCTO. The micrograph images (Figure 3(b)) show that the CCTO grains do not present a perfect spherical geometry, and the discrepancy between experimental and predicted values could be directly related to this fact.

Since CCTO particles dispersed in the matrix have no spherical shape, as could be evidenced by SEM, the models that take into account different morphologies for the

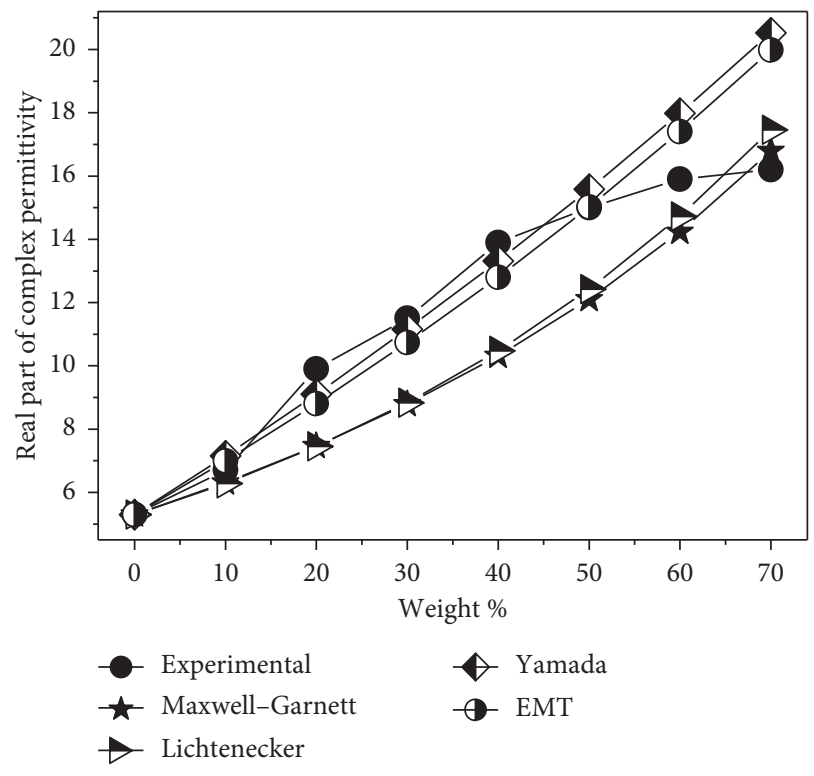

Figure 6: Real part of complex permittivity of the composite samples, as a function of the weight fraction of CCTO, obtained for the $5 \mathrm{GHz}$ cavity, and respective fitting using four classic mixing models.

particles are more suitable. One example of this is the effective medium theory (EMT) model. The real part of the dielectric constant for the composite in the EMT model is given as follows [34]:

$$
\mathcal{\varepsilon}^{\prime}=\varepsilon_{1}\left(1+\frac{V_{\mathrm{f}}\left(\varepsilon_{2}-\varepsilon_{1}\right)}{\varepsilon_{1}-n\left(1-V_{\mathrm{f}}\right)\left(\varepsilon_{2}-\varepsilon_{1}\right)}\right),
$$

where $n$ is a ceramic morphology fitting factor. Small values of $n$ indicate the filler particles have a shape nearly spherical, while high values of $n$ indicate that the shape of the particles is very irregular. The better value for the shape parameter obtained for the experimental results was $n=0.1$. Although this model is suitable for particles with dimensions less than $1 \mu \mathrm{m}$, Figure 6 shows a good approximation between the values predicted by the EMT model and the experimental data obtained for the composites with a weight fraction up to $50 \%$. However, for higher weight fractions, the model begins to deviate, which should be related to an increasing aggregation of the CCTO particles as the polymer matrix becomes less significant.

From Figure 3(b), it can be seen that the CCTO grain morphology and granulometry are not homogenous for all the particle distribution, presenting differences in size and shape. In addition, from Figure 3(d), one can note that some irregular dispersion of the CCTO particles in the polymeric matrix is present. Thereby, another model was used to try a better agreement with the experimental curve: the Yamada model. The Yamada model [35] predicts the following values for real part of the dielectric constant of a biphasic composite:

$$
\varepsilon^{\prime}=\varepsilon_{1}\left(1+\frac{\gamma V_{\mathrm{f}}\left(\varepsilon_{2}-\varepsilon_{1}\right)}{\gamma \varepsilon_{1}-\left(1-V_{\mathrm{f}}\right)\left(\varepsilon_{2}-\varepsilon_{1}\right)}\right),
$$


where $\gamma$ is also a fitting factor associated with the ceramic grain morphology. The best fitting was obtained for $\gamma=$ 14.309. Globally, the fitting performed with the EMT model presents a better approximation to the experimental data. However, both Yamada and EMT models fail to predict the dielectric behavior of these CCTO/PVA composites for weight fractions higher than $50 \%$. Once again, this discrepancy should be related to a deficient dispersion of the minor phase.

\section{Conclusions}

Two resonant cavities with $2.7 \mathrm{GHz}$ and $5.0 \mathrm{GHz}$ resonant frequencies were used to measure the complex permittivity of polymer matrix composites, prepared by the dispersion of CCTO $\left(\mathrm{CaCu}_{3} \mathrm{Ti}_{4} \mathrm{O}_{12}\right)$ powders, prepared by the solid-state method, in a polyvinyl alcohol (PVA) matrix. Different weight fractions of the filler were used to form a flexible and high-dielectric constant composite at GHz region. PVA was chosen as the polymeric matrix because of its high thermal and chemical stability, nontoxicity, and for presenting a high-dielectric strength and good charge storage capacity, which makes it usable as the polymer matrix in compositebased electronic components. Within this study, we were able to prepare, by a simple method, a ceramic/polymer composite with desirable dielectric properties, particularly at the microwave frequency region, such as a high-dielectric constant and low dissipation factor. As expected, the dielectric constant increased with the weight fraction of CCTO for both analyzed frequencies, and a similar behavior is observed for $\varepsilon^{\prime \prime}$. In an attempt to predict the dielectric constant of the CCTO/PVA composites as a function of the weight fraction of the filler, several classical mixing models were used. This way, the EMT (with $n=0.1$ ) and Yamada (with $\gamma=14.309$ ) models were identified as the most suitable to fit the experimental data. Best fitting results, using EMT and Yamada models, were obtained for weight fractions lower than 50\%. Both EMT and Yamada models are more associated with nonspherical shape particles dispersed in a matrix, a fact that could be evidenced by the SEM.

\section{Data Availability}

The data used to support the findings of this study are available from the corresponding author upon request.

\section{Disclosure}

An earlier version has been presented as a poster at the First International Symposium on Dielectric Materials and Applications (ISyDMA'2016).

\section{Conflicts of Interest}

The authors declare that they have no conflicts of interest.

\section{Acknowledgments}

We thank CAPES Brazilian agency (Coordenação de Aperfeiçoamento de Pessoal de Nível Superior) for the scholarship of K. D. A. Sabóia. This work was funded by FEDER funds through the COMPETE 2020 Programme and National Funds through FCT-Portuguese Foundation for Science and Technology under the project UID/CTM/ 50025/2013.

\section{Supplementary Materials}

(1a) Porosity of the sample PVA (resume); (1b) porosity of the sample PVA (full data); (2a) porosity of the sample $\mathrm{CCTO}(20)$ (resume); (2b) porosity of the sample CCTO(20) (full data); (3a) porosity of the sample CCTO(50) (resume); (3b) porosity of the sample CCTO(50) (full data); (4) micrograph of the PVA sample with representation of pores (red); (5) micrograph of $\mathrm{CCTO}(20)$ with representation of pores (red); (6) micrograph of $\mathrm{CCTO}(50)$ with representation of pores (red). (Supplementary Materials)

\section{References}

[1] P. Hu, Y. Shen, Y. Guan et al., "Topological-structure modulated polymer nanocomposites exhibiting highly enhanced dielectric strength and energy density," Advanced Functional Materials, vol. 24, no. 21, pp. 3172-3178, 2014.

[2] Z. B. Pan, L. M. Yao, J. W. Zhai, D. Z. Fu, B. Shen, and H. T. Wang, "High-energy-density polymer nanocomposites composed of newly structured one-dimensional $\mathrm{BaTiO}_{3} @$ $\mathrm{Al}_{2} \mathrm{O}_{3}$ nanofibers," ACS Applied Materials \& Interfaces, vol. 9, no. 4, pp. 4024-4033, 2017.

[3] B. Xie, H. B. Zhang, Q. Zhang et al., "Enhanced energy density of polymer nanocomposites at a low electric field through aligned $\mathrm{BaTiO}_{3}$ nanowires," Journal of Materials Chemistry A, vol. 5, no. 13, pp. 6070-6078, 2017.

[4] Y. Mobarak, M. Bassyouni, and M. Almutawa, "Materials selection, synthesis, and dielectrical properties of PVC nanocomposites," Advances in Materials Science and Engineering, vol. 2013, Article ID 149672, 6 pages, 2013.

[5] Y. H. Jin, N. Xia, and R. A. Gerhardt, "Enhanced dielectric properties of polymer matrix composites with $\mathrm{BaTiO}_{3}$ and MWCNT hybrid fillers using simple phase separation," Nano Energy, vol. 30, pp. 407-416, 2016.

[6] F. Amaral, C. P. L. Rubinger, F. Henry, L. C. Costa, M. A. Valente, and A. Barros-Timmons, "Dielectric properties of polystyrene-CCTO composite," Journal of Non-Crystalline Solids, vol. 354, pp. 5321-5322, 2008.

[7] Y. Shen, A. Gu, G. Liang, and L. Yuan, "High performance $\mathrm{CaCu}_{3} \mathrm{Ti}_{4} \mathrm{O}_{12} /$ cyanate ester composites with excellent dielectric properties and thermal resistance," Composites Part A: Applied Science and Manufacturing, vol. 41, no. 11, pp. 1668-1676, 2010.

[8] H. N. Friedlander, H. E. Harris, and J. G. Pritchard, "Structure-property relationships of poly(vinyl alcohol). I. Influence of polymerization solvents and temperature on the structure and properties of poly(vinyl alcohol) derived from poly(vinyl acetate)," Journal of Polymer Science Part A-1: Polymer Chemistry, vol. 4, no. 3, pp. 649-664, 1966.

[9] S. K. Pasha, K. Deshmukh, M. B. Ahamed, K. Chidambaram, M. K. Mohanapriya, and N. A. Raj, "Investigation of microstructure, morphology, mechanical, and dielectric properties of PVA/PbO nanocomposites," Advances in Polymer Technology, vol. 36, no. 3, pp. 352-361, 2017.

[10] K. Deshmukh, M. B. Ahamed, R. R. Deshmukh et al., "Synergistic effect of vanadium pentoxide and graphene oxide 
in polyvinyl alcohol for energy storage application," European Polymer Journal, vol. 76, pp. 14-27, 2016.

[11] M. A. Subramanian, D. Li, N. Duran, B. A. Reiner, and A. W. Sleight, "High dielectric constant in $\mathrm{ACu}_{3} \mathrm{Ti}_{4} \mathrm{O}_{12}$ and $\mathrm{ACu}_{3} \mathrm{Ti}_{3} \mathrm{FeO}_{12}$ phases," Journal of Solid State Chemistry, vol. 151, no. 2, pp. 323-325, 2000.

[12] M. S. Ivanov, F. Amaral, V. A. Khomchenko, J. A. Paixão, and L. C. Costa, "Investigation of micro- and nanoscale barrier layer capacitance mechanisms of conductivity in $\mathrm{CaCu}_{3} \mathrm{Ti}_{4} \mathrm{O}_{12}$ via scanning probe microscopy technique," RSC Advances, vol. 7, no. 65, pp. 40695-40704, 2017.

[13] S. Jesurani, S. Kanagesan, S. Hashim, I. Ismail, and I. Ibrahim, "Microstructural and dielectric properties of $\mathrm{Zr}$ doped microwave sintered $\mathrm{CaCu}_{3} \mathrm{Ti}_{4} \mathrm{O}_{12}$ synthesized by sol-gel route," Advances in Materials Science and Engineering, vol. 2014, Article ID 187420, 6 pages, 2014.

[14] F. Amaral, L. C. Costa, M. A. Valente et al., "Colossal dielectric constant of poly-and single-crystalline $\mathrm{CaCu}_{3} \mathrm{Ti}_{4} \mathrm{O}_{12}$ fibers grown by the laser floating zone technique," Acta Materialia, vol. 59, no. 1, pp. 102-111, 2011.

[15] M. A. Subramanian, W. J. Marshall, T. G. Calvarese, and A. W. Sleight, "Valence degeneracy in $\mathrm{CaCu}_{3} \mathrm{Cr}_{4} \mathrm{O}_{12}$," Journal of Physics and Chemistry of Solids, vol. 64, no. 9-10, pp. 1569-1571, 2003.

[16] S. V. Kalinin, J. Shin, G. M. Veith et al., "Real space imaging of the microscopic origins of the ultrahigh dielectric constant in polycrystalline $\mathrm{CaCu}_{3} \mathrm{Ti}_{4} \mathrm{O}_{12}$," Applied Physics Letters, vol. 86, no. 10, pp. 102902-102905, 2005.

[17] F. Amaral, C. P. L. Rubinger, M. A. Valente, L. C. Costa, and R. L. Moreira, "Enhanced dielectric response of $\mathrm{GeO}_{2}$-doped $\mathrm{CaCu}_{3} \mathrm{Ti}_{4} \mathrm{O}_{12}$ ceramics," Journal of Applied Physics, vol. 105, no. 3, p. 034109, 2009.

[18] A. P. Ramirez, M. A. Subramanian, M. Gardel et al., "Giant dielectric constant response in a copper-titanate," Solid State Comunications, vol. 115, no. 5, pp. 217-220, 2000.

[19] X. You, N. Chen, and G. Du, "Constructing threedimensionally interwoven structures for ceramic/polymer composites to exhibit colossal dielectric constant and high mechanical strength: $\mathrm{CaCu}_{3} \mathrm{Ti}_{4} \mathrm{O}_{12}$ /epoxy as an example," Composites Part A: Applied Science and Manufacturing, vol. 105, pp. 214-222, 2018.

[20] Z. M. Dang, T. Zhou, S. H. Yao et al., "Advanced calcium copper titanate/polyimide functional hybrid films with high dielectric permittivity," Advanced Materials, vol. 21, no. 20, pp. 2077-2082, 2009.

[21] P. Mishra and P. Kumar, "Enhancement of dielectric properties of 0.2 [BZT-BCT] $-0.8[(1-x)$ epoxy- $x$ CCTO $](x=$ $0.02,0.04,0.06,0.08 \& 0.1$ ) composites for embedded capacitor and energy harvesting applications," Journal of Alloys and Compounds, vol. 617, pp. 899-904, 2014.

[22] X. L. Chao, P. Wu, Y. Zhao, P. F. Liang, and Z. P. Yang, "Effect of $\mathrm{CaCu}_{3} \mathrm{Ti}_{4} \mathrm{O}_{12}$ powders prepared by the different synthetic methods on dielectric properties of $\mathrm{CaCu}_{3} \mathrm{Ti}_{4} \mathrm{O}_{12} /$ polyvinylidene fluoride composites," Journal of Materials Science: Materials in Electronics, vol. 26, no. 5, pp. 3044-3051, 2015.

[23] B. Shri Prakash and K. B. R. Varma, "Dieletric behavior of CCTO/epoxy and Al-CCTO/epoxy composites," Composites Science and Technology, vol. 67, no. 11-12, pp. 2363-2368, 2007.

[24] A. G. Pinheiro, F. M. Pereira, M. R. P. Santos, H. H. B. Rocha, and A. S. B. Sombra, "Electric properties of $\mathrm{Bi}_{4} \mathrm{Ti}_{3} \mathrm{O}_{12}(\mathrm{BIT})$ $\mathrm{CaCu}_{3} \mathrm{Ti}_{4} \mathrm{O}_{12}$ (CCTO) composite substrates for high dielectric constant devices," Journal of Materials Science, vol. 42, no. 6, pp. 2112-2120, 2007.
[25] A. F. L. Almeida, P. B. A. Fechine, J. C. Góes, M. A. Valente, M. A. R. Miranda, and A. S. B. Sombra, "Dielectric properties of $\mathrm{BaTiO}_{3}$ (BTO) - $\mathrm{CaCu}_{3} \mathrm{Ti}_{4} \mathrm{O}_{12}$ (CCTO) composite screenprinted thick films for high dielectric constant devices in the medium frequency (MF) range," Materials Science and Engineering B, vol. 111, no. 2-3, pp. 113-123, 2004.

[26] L. A. Ramajo, M. A. Ramírez, P. B. Bueno, M. M. Reboredo, and M. S. Castro, "Dielectric behaviour of $\mathrm{CaCu}_{3} \mathrm{Ti}_{4} \mathrm{O}_{12}$-epoxy composites," Materials Research, vol. 11, no. 1, pp. 85-88, 2008.

[27] M. Abdelaziz and E. M. Abdelrazek, "Effect of dopant mixture on structural, optical and electron spin resonance properties of polyvinyl alcohol," Physica B: Condensed Matter, vol. 390, no. 1-2, pp. 1-9, 2007.

[28] K. D. Sabóia, H. Girão, A. Sombra et al., "Microwaves dielectric properties of $\mathrm{Y}_{3} \mathrm{Fe}_{5} \mathrm{O}_{12}-\mathrm{CaCu}_{3} \mathrm{Ti}_{4} \mathrm{O}_{12}$ composites," in Proceedings of IEEE International Conference in Microwave and Optoelectronics (IMOC'11), pp. 537-540, Natal, Brazil, October 2011.

[29] L. C. Costa, S. Devesa, and F. Henry, "A 5 GHz resonant cavity for complex permittivity measurements: design, test performances and application," Materials Science Forum, vol. 514516, pp. 1561-1565, 2006.

[30] J. Laugier and A. Filhol, CELREF Program, ILL, Grenoble, France, 1978.

[31] J. C. Maxwell, A Treatise on Electricity and Magnetism, Dover Corporation, Downers Grove, IL, USA, 1954.

[32] S. George and M. T. Sebastian, "Three-phase polymerceramic-metal composite for embedded capacitor applications," Composites Science and Technology, vol. 69, no. 7-8, pp. 1298-1302, 2009.

[33] H. S. Nalwa, Ferroelectric Polymers, Marcel Dekker, New York, NY, USA, 1995.

[34] Y. Rao, J. Qu, T. Marinis, and C. P. Wong, "A precise numerical prediction of effective dielectric constant for polymerceramic composite based on effective-medium theory," IEEE Transactions on Components and Packaging Technologies, vol. 23, no. 4, pp. 680-686, 2000.

[35] T. Yamada, T. Ueda, and T. Kitayama, "Piezoelectricity of a high-contne lead zirconate titanate/polymer composite," Journal of Applied Physics, vol. 53, no. 6, pp. 4328-4332, 1982. 


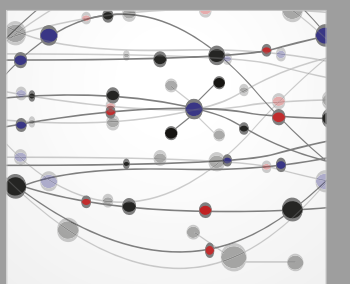

The Scientific World Journal
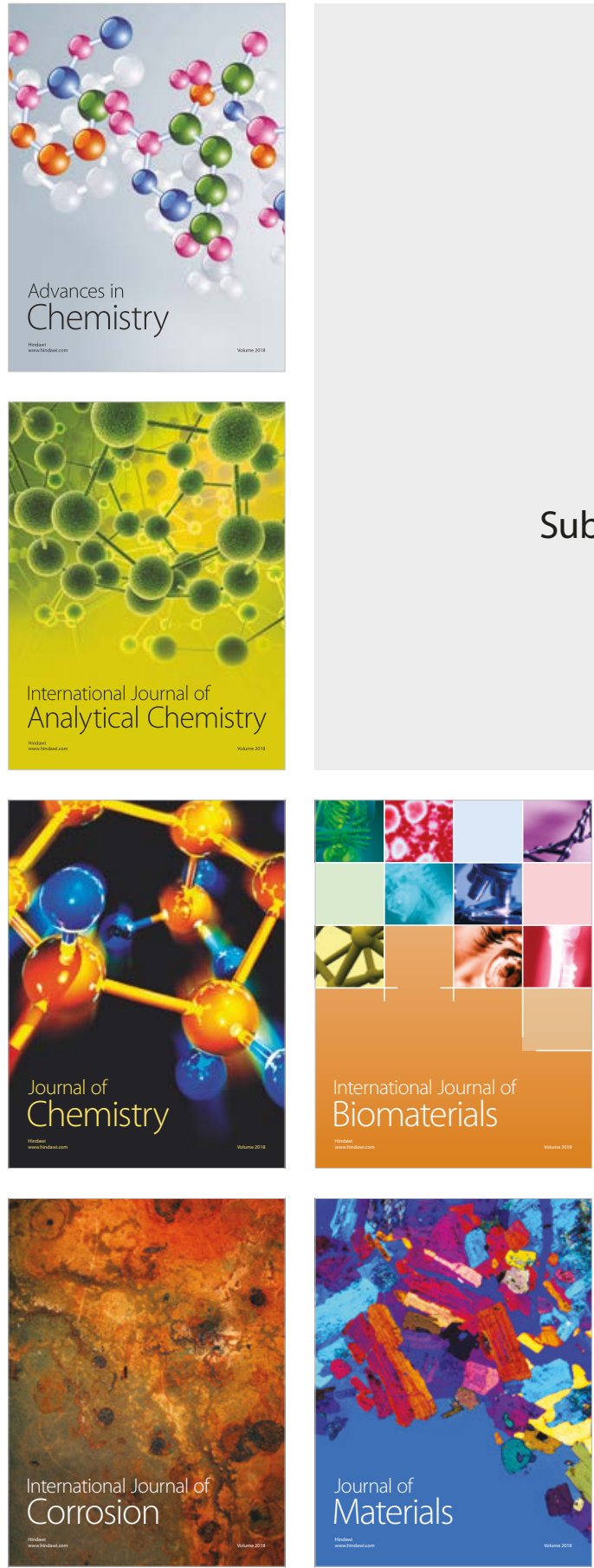

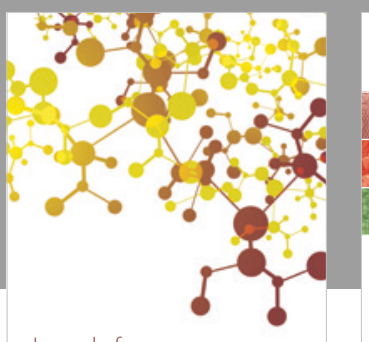

Journal of

Applied Chemistry
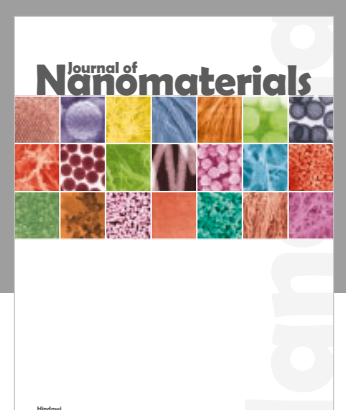

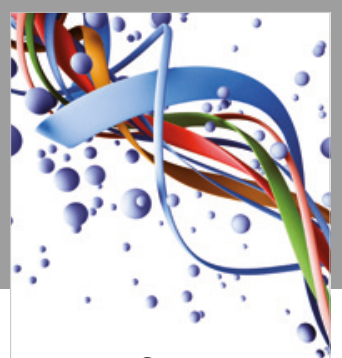

Scientifica

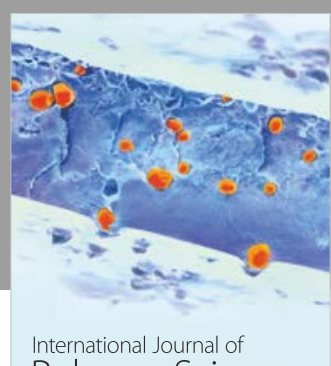

Polymer Science

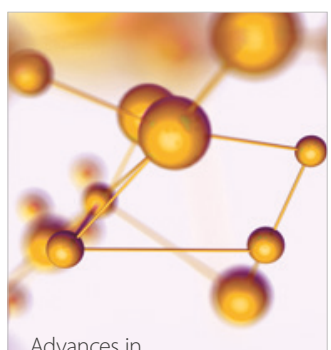

Physical Chemistry
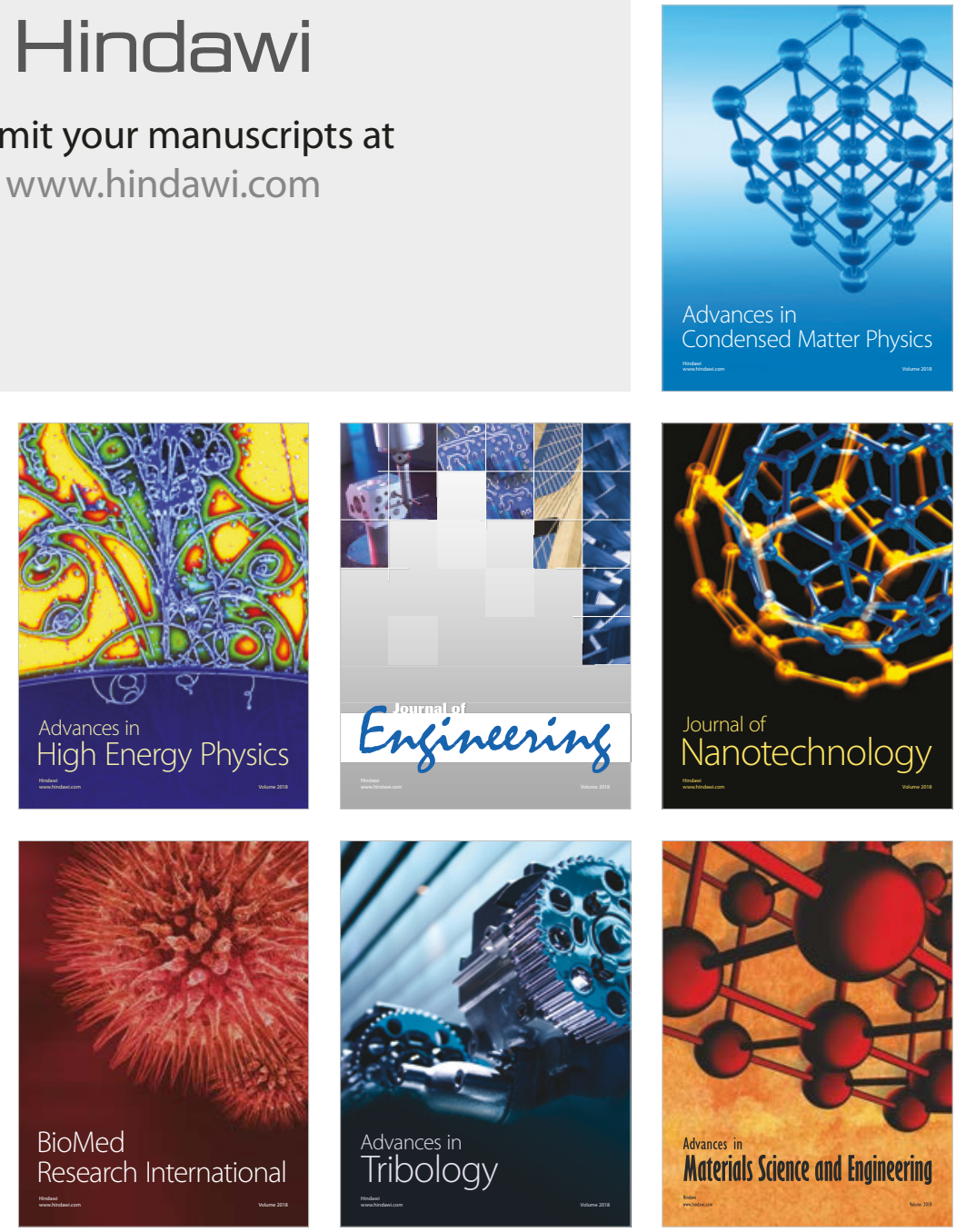